\title{
Evidence of Kosterlitz-Thouless phase transitions in the Ising model with dipolar interactions
}

\author{
M. A. Bab (1) and G. P. Saracco \\ Instituto de Investigaciones Fisicoquímicas Teóricas y Aplicadas (INIFTA), UNLP, CCT La Plata-CONICET, \\ c.c. 16, Suc. 4, (1900)La Plata, Argentina
}

(Received 20 February 2019; published 29 August 2019)

\begin{abstract}
The ferromagnetic bidimensional Ising model with dipolar interactions has been proposed to model ultrathin films with strong out-of-plane anisotropy. The phase diagram presents a rich phenomenology that includes lowtemperature phases characterized by stripes of width $n\left(h_{n}\right)$ and a high-temperature phase with domains of stripes with mutually perpendicular orientations, named tetragonal liquid (TL). The latter phase can be reached by two possible ways. One of them is the direct transition $h_{n}$ to TL, and the other one is through an intermediate phase with orientational order but short-range positional disorder, named nematic phase (NM). The regions of the phase diagram where these transitions occur, as well as their character, remain an open question and are the object of the present work. In order to clarify this topic, intensive Monte Carlo simulations were performed by employing short-time dynamics as the main tool for studying the phase transition behavior. The dynamic evolution of the orientational order parameter and its moments are measured for selected values of the ratio between the ferromagnetic exchange and dipolar constants, called $\delta$. The obtained results indicate that the intermediate NM phase is present for $\delta \geqslant 2$ in narrow ranges of temperatures. Also, the results suggest that both transitions, i.e., $h_{n}$-NM and NM-TL, have a Kosterlitz-Thouless character. This type of topological transition is observed in continuous bidimensional models and have been proposed for discrete ones, as in the case of the present work.
\end{abstract}

DOI: 10.1103/PhysRevE.100.022143

\section{INTRODUCTION}

In the last years, thin magnetic films have been a subject of growing interest from both the theoretical and experimental points of view. This is mainly motivated by a rich phenomenology that includes giant magnetoresistance [1], tunneling magnetoresistance [2] and exchange bias [3], as well as by technological applications in spintronic devices [4,5], microwave antennas [6], tunable inductors [7], recording media, and magnetic field sensors [8-11]. These applications depend crucially on the reliable control of the magnetization orientation. Therefore, the comprehension of the involved physics mechanisms remains a high priority.

By varying the composition and thickness of the films, it is possible to build them in such a way that strongly favors the perpendicular orientation of the magnetization. In this way, it has been found that ultrathin films with perpendicular magnetic anisotropy present nonplanar domain structures, even if the shape anisotropy is dominant [12,13]. In this last case, a magnetization reorientation transition where the magnetization changes from planar to nonplanar occurs and depends on both the film thickness [13-15] and the temperature of the thermal bath [15-17]. In the range of temperatures and thicknesses where the magnetization has an out-of-plane component, the competition between spin-spin dipolar interactions and surface anisotropy leads to stripe patterns that define regions with alternate opposite directions [15,18]. These domain structures have been observed in several metallic ferromagnetic films, such as $\mathrm{Co}$ [19], $\mathrm{Fe}$ [20-22], $\mathrm{Fe}_{20} \mathrm{Ni}_{80}$ [23], FePt [12], and $\mathrm{CoPb}$ [24], among others.

Motivated by these results, several theoretical approaches have been proposed. In particular, the magnetic behavior of materials in the limit of the strong perpendicular anisotropy has been modeled by the well-known planar Ising model with short-range ferromagnetic and long-range dipolar interactions, i.e.,

$$
\mathcal{H}=-\delta \sum_{\langle i ; j\rangle} \sigma_{i} \sigma_{j}+\sum_{i<j} \frac{\sigma_{i} \sigma_{j}}{r_{i j}^{3}},
$$

where $\sigma_{i}= \pm 1$ is the Ising spin variable oriented perpendicularly to the square lattice of size $L$, and $\delta=J / g$ is the ratio between the short-range exchange $(J>0)$ and the long-range dipolar $(g>0)$ constants. Also, the first sum runs over all pairs of nearest-neighbor (NN) spins, while the second one runs over all pairs of spins $(i, j)$ of the lattice separated by a distance $r_{i j}$, measured in crystal units. The energy is measured in units of $g$. Since the ferromagnetic order is destroyed by the frustration originated by the dipolar interactions, an interesting but complex phase diagram comes up. The $T-\delta$ phase diagram, where $T$ is the temperature of the thermal bath, has been widely studied analytically $[15,18,25,26]$, and by Monte Carlo simulations [27-34]. The obtained results showed that both the ground-state $(T=0)$ [26] and the lowtemperature regimes from $\delta>0.4403$ present domain structures characterized by stripes of an integer width $n$ measured in lattice units $\left(h_{n}\right)$, which run along one axis of the lattice where the model is set. The stripe width $n$ increases with $\delta$ always in consecutive integer numbers. If $T$ is raised enough, then the equilibrium state corresponds to a disordered phase with a discrete fourfold rotational symmetry called tetragonal liquid (TL), where stripes of mutually perpendicular orientations emerge and form labyrinthine patterns. In turn, this phase changes continuously to a paramagnetic phase in the high-temperature limit for all $\delta$ values. At intermediate temperatures, two different scenarios have been reported, in 
one of them the system has a transition $h_{n}$-TL as the temperature rises $[25,29]$. In the second scenario an intermediate phase appears, which is characterized by long-range orientational order but no long-range positional order with band dislocations and disclinations, named nematic phase (NM). Until now, the NM phase has been observed only in narrow windows around $\delta=2.2$ and $\delta=2.8[29,31,35]$.

The $h_{n}$-TL phase transitions have been the focus of a longstanding controversy mainly related to the order of the transitions and their extension [28-30,32,36]. This controversy is a consequence of the limitations introduced by the long-range dipolar interactions on the Monte Carlo simulation results, as will be discussed in the following section. Recently, by using the short-time dynamics approach (STD), Horowitz et al. [33] showed that the $h_{1}$-TL transition line presents a continuous character, in agreement with the results obtained by Fonseca et al. [32] by studying the complex partition function zeros from multicanonical algorithms. Later, Bab et al. studied the $h_{2}$-TL transition line and demonstrated that it is of first order for the $1.2585 \leqslant \delta \leqslant 1.36$ interval and continuous for the $1.37 \leqslant \delta \leqslant 1.9$ interval, with a tricritical point at $\delta \lesssim 1.37$. Also it was found that $\delta=1.2585$ is a coexistence point of the $h=1, h=2$ and TL phases [34].

On the other hand, the results presented by Abanov et al. [25], obtained by analyzing a continuous approximation to the domain wall crystal, predicted the existence of a Kosterlitz-Thouless (KT) phase transition type between $h_{n}$ and NM phases and, at higher temperatures, the transition to TL phase. Cannas et al. have thoroughly studied the model for $\delta=2$ [35] by Monte Carlo simulations verifying the existence of a stable NM phase. The nature of the phase transitions $h_{2}$-NM-TL was analyzed by studying the behavior of the energy, specific heat, fourth-order Binder's cumulant and the susceptibility of the orientational order parameter [35] in the equilibrium regime. By performing a finite-size scaling of the specific heat and the cumulant, they concluded that the $h_{2}$-NM transition is of first order. However, this result cannot be taken as definitive, since they also found other features that suggest a KT transition, such as the continuity of the energy per spin and the saturation in the specific heat maximum as the system size increases. Similar discrepancies were reported by Rizzi and Alves [31] by employing histogram reweighting techniques. With respect to the NM-TL the results were not conclusive, and the possibility that the transition is KT cannot be excluded $[31,35]$.

In view of the above-commented works and their difficulties in giving a correct interpretation of the obtained data, the goal of this work will be to characterize both the $h_{n}$-NM and the NM-TL phase transitions as well as the existence of NM phase in other intervals of the phase diagram by means of STD. The case $\delta=2$ will be carefully studied and then extended to other values of $\delta$. This work is organized as follows: In Sec. II the simulation details and a summary of the STD method are given; in Sec. III the results are presented and discussed; and, finally, the conclusions are reported in Sec. IV.

\section{SHORT-TIME DYNAMICS AND SIMULATION DETAILS}

As was mentioned, long-range dipole interactions are responsible for the shortcomings in the characterization of the nature of the phase transitions among $h_{n}$, NM, and TL. In fact, they originate a limitation in the lattice sizes employed in order to have reasonable simulation times to reach equilibrium states, especially in the phase transition zone. So finite-size effects are very strong, and the application of the finite-size scaling theory in the determination of the critical exponents becomes a hard task to perform. Moreover, the presence of multiple metastable states at low temperatures requires very long time intervals for equilibration, so any weak first-order transition may be misinterpreted as a continuous transition.

With an aim to avoid these hindrances, the short-time dynamics approach has already been successfully applied to this model $[33,34]$. In this method, it is not necessary to have a system in equilibrium, and the simulations proceed within a short-time interval $\left(t_{\operatorname{mic}}, t_{\max }\right)$. In this interval, the dynamic behavior of the physical observables follows STD scaling laws. Here $t_{\text {mic }}$ and $t_{\max }$ are set when the spatial correlation length $[\xi(t)]$ is of the order of lattice spacing and the system size $(L)$, respectively. As a consequence, STD is free of the critical slowdown due to the fact that $t_{\max }$ is shorter than the equilibration time. The STD consists on the analysis of the dynamic evolution of the order parameter and its moments in a neighborhood of the transition points for different values of the control parameter. In the present work, the control parameter is the temperature $(T)$ and the order parameter is that introduced by Booth et al. [37],

$$
O_{h v} \equiv \frac{n_{v}-n_{h}}{n_{v}+n_{h}}
$$

where $n_{v}\left(n_{h}\right)$ is the number of vertical (horizontal) bonds of the NN antiparallel spins. This definition assures that $O_{h v}=+1(-1)$ when the system is in the stripe horizontal (vertical) ordered phase, and $O_{h v}=0$ in the TL or paramagnetic phases. The susceptibility of the orientational order parameter is defined as:

$$
\chi=N\left(\left\langle O_{h v}^{2}\right\rangle-\left\langle O_{h v}\right\rangle^{2}\right)
$$

where $N=L^{2}$ is the number of spins and $\langle\ldots\rangle$ indicates the average over different thermal histories from equivalent initial conditions. For continuous transitions and at the critical point $\left(T=T_{c}\right)$, the order parameter and its moments exhibit a power-law behavior within the short-time regime when the system is initialized from the ground state or the paramagnetic one at $T=0$ or $T=\infty$, respectively [38,39]. The power-law exponents are related to the critical exponents defined at equilibrium. For $T \neq T_{c}$, but at the neighborhood of the critical point, these power laws are modified by scaling functions. This fact allows us to determine the critical temperature as well as the critical exponents from the localization of the best power law (for more details, see the review in Ref. [38] and references therein).

For the case of ground-state initial condition, the time evolution of the observables is the following:

$$
\begin{gathered}
O_{h v}(t) \propto t^{-v}, \quad v=\beta / \nu z, \\
\chi(t) \propto t^{\mu}, \quad \mu=\gamma / \nu z .
\end{gathered}
$$


However, if the system is started from paramagnetic initial condition, then the proposed scaling law is

$$
O_{h v}^{2}(t)=\chi(t) / N \propto t^{\mu}
$$

where $\beta, v$, and $\gamma$ are the static critical exponents for the order parameter, correlation length, and susceptibility, respectively; and $z$ the dynamic exponent for the time evolution of the spatial correlation length. For the first-order phase transitions, the susceptibility diverges as power-law behavior at the metastability limits of the coexisting phases, which are identified as the spinodal points $[38,40]$. Furthermore, if the system is started from the ground-state condition, then the spinodal value of the order parameter $O_{h v}^{\mathrm{sp}}$ must be taken into account, so $O_{h v}(t)-O_{h v}^{\mathrm{sp}}$ diverges as a power law. The difference between the spinodal points temperatures is a measure of the strength of the transition and it allows us to distinguish between a continuous and a weak first-order transition [38].

The STD approach has also been used to characterize the KT transition in models with short-range interactions such as the XY model [41-43], its fully frustrated version [44], and the six-state clock model [45]. It is well known that in a KT phase transition, the dynamic evolution of the order parameter and its moments follows a power law, with exponents depending on the temperature, for $T \leqslant T_{\mathrm{KT}}$, and the usual exponential decay for $T>T_{\mathrm{KT}}[38,43,45]$. In this way, the system is said to remain critical for $T \leqslant T_{\mathrm{KT}}$. This fact hinders the estimation of the error bars in the lower bound, i.e., for $T<T_{\mathrm{KT}}$. In order to overcome this difficulty, the nonequilibrium relaxation method proposed by Ozeki et al. [39] can be applied to study the dynamic behavior of the observables in the $T>T_{\mathrm{KT}}$ interval from the ground-state initial configuration. Based on this method it is expected that the order parameter, $O_{h v}$, will scale as:

$$
O_{h v}(t, \epsilon)=\tau^{-\lambda} O^{\prime}[t / \tau(\epsilon)],
$$

where $\lambda$ is a dynamic exponent assumed to be temperature independent, $\epsilon=\left(T-T_{\mathrm{KT}}\right) / T_{\mathrm{KT}}$ is the reduced temperature, $O^{\prime}[t / \tau(\epsilon)]$ is a scaling function, and $\tau$ is the relaxation time. It is expected that $\tau$ will diverge exponentially with $\epsilon$ in the same way as the correlation length $\left[\xi=\xi_{0} e^{\left(c / \epsilon^{v}\right)}\right]$, i.e.,

$$
\tau=a e^{\left(b / \epsilon^{\nu}\right)} .
$$

Finally, let us recall that for models with short-range interactions, STD is free of finite-size effects since $\xi(t) \ll L$. This is no longer valid for long-range interactions and when periodic boundary conditions are implemented by introducing a cutoff in the interaction sum. This cutoff affects the temperature and the range where the power-law behavior is observed but not the critical exponents [46]. In order to properly address this problem, periodic boundary conditions were implemented by means of the sum over replicas. In this method, the entire space is tiled with replicas of the original finite system, forming an aggregation of size $m$ in units of replicas. In this way, the interactions in the Hamiltonian of Eq. (1) are replaced by effective interactions that take into account not only the interactions of the original system but also the interactions with its replicas [33].

Monte Carlo simulations were performed in square lattices with sizes $L$ by using the Metropolis algorithm with heat bath dynamics. In order to avoid the introduction of an artificial frustration, the system size has to be commensurate with $h$ for each $\delta$, so $L=120$ was chosen for $h=3,5$ and $L=128$ for $h=2,4$. With the aim to assure that the determined transition temperatures correspond to those of the thermodynamic limit, a previous study for different system and aggregation sizes was carried out at selected temperatures (not shown for the sake of space). It was found that for systems with $L>100, m=5000$ was determined as enough in order to obtain results that are independent of $L$ within the error bars. From these results, the range of validity of the STD method was obtained by searching the longest time interval where the susceptibility exhibited the best power-law behavior defined by means of the local exponent [33]. In this way, $t_{\text {mic }} \leqslant 400$ and $t_{\max }>5000$ MCS were estimated for the studied $\delta$ and temperature values. The STD observables were averaged over 2000 different realizations.

\section{RESULTS AND DISCUSSION}

In Sec. I, results about the existence of the NM phase in narrow intervals $T_{1}<T<T_{2}$ around the $h_{2}-h_{3}$ and $h_{3}-h_{4}$ transition lines were mentioned. They confirmed the existence of the second scenario predicted by Abanov [25,29], where two phase transitions were observed, i.e., $h_{n}$-NM and NM-TL. In the present work, the STD method was applied to study these intervals and extended up to $\delta=3.6$ where the ground state corresponds to the $h_{5}$ stripe phase.

\section{A. Phase transitions for $\delta=2$}

First, the STD results for the case $\delta=2$ will be described in detail. This is a good starting point because it has been investigated extensively [29,31,35,47], so our results can be directly compared with the previous findings. Figure 1 shows the time evolution of the second moment of the orientational order parameter, $O_{h v}^{2}$, when the system of lattice size $L=128$ is quenched from the high-temperature paramagnetic phase-corresponding to the configurations at $T=\infty$-up to the indicated temperatures. As can be observed in the main panel, the data follow a power-law time evolution, as expected in a critical point, but this behavior is also observed for all quenching temperatures in the interval $0.60 \leqslant T \leqslant 0.8 .05$. This interval includes the already reported transition temperatures, $T_{1} \approx 0.77$ and $T_{2} \approx 0.80$ for $h_{2}$-NM and NM-TL phase transitions, respectively $[31,35]$. The inset shows the departure from the power-law behavior observed for temperatures $T>0.805$, therefore this value can be assumed as the NM-TL transition point in excellent agreement with the reported value $T_{2}$. Since power-law behavior is expected only at the temperature corresponding to a critical or paramagnetic spinodal point for the cases of continuous or first-order phase transitions, respectively (see Sec. II), the obtained results allow us to rule out both kinds of transitions at $T=0.805$.

Due to fact that the phenomenology of the Ising dipolar model reveals itself only for rather large lattice sizes, controversial findings from finite-size scaling have been reported about this transition point. On the one hand, for system sizes in the interval $32 \leqslant L \leqslant 64$, a weak first order was proposed [35] in view of the obtained results of the scaling of the response functions (specific heat and susceptibility) and 


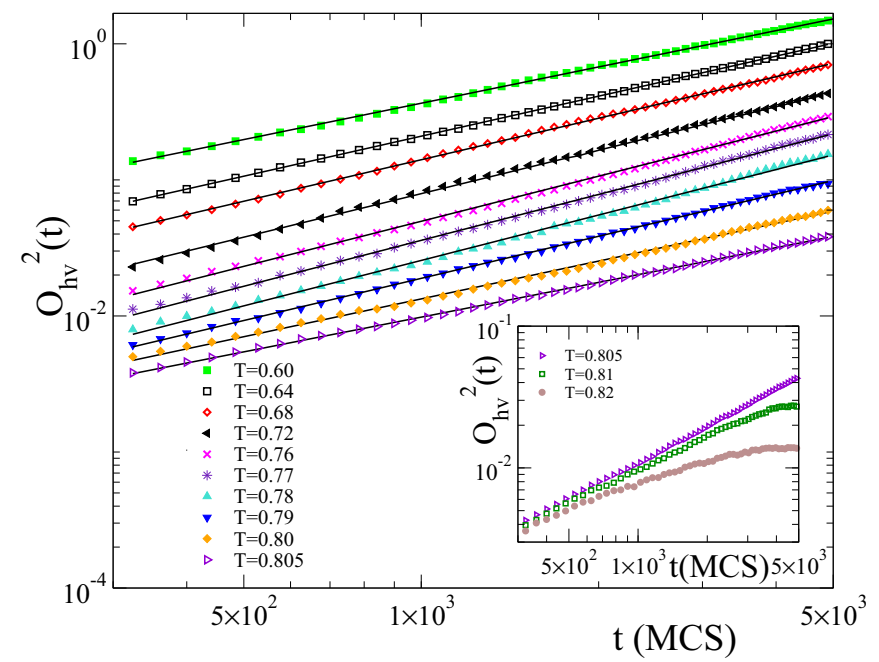

FIG. 1. Log-log plot of the $O_{h v}^{2}$ time evolution from the paramagnetic initial condition at the indicated temperatures. The system size and interaction parameter used are $L=128$ and $\delta=2$, respectively. Due to superposition of the curves for different temperatures, the data were shifted by a multiplicative factor with the aim of clearly showing their power-law behavior. The inset exhibits the original data (i.e., they are not multiplied by a factor) corresponding to the temperatures at which departures from the power law are observed. For the sake of comparison, $T=0.805$ is also included.

cumulant minimum. On the other hand, from the study of the same response functions in systems of sizes $32 \leqslant L \leqslant 72$, exponents compatible with a continuous phase transition were obtained [31]. However, in both works the hypothesis of a KT transition was also conjectured, based on the absence of divergences in the specific heat. The present work supports this last hypothesis, in view that the power-law behavior suggests a critical character for $T \leqslant 0.805$. Furthermore, if the theoretical values of $v$ and $\eta$ predicted for the KT transition, i.e., $v=0.5$ and $\eta=0.25$, are employed in the hyperscaling relationship $\gamma / v=(d-\eta)$, then the dynamical critical exponent $z$ can be estimated for the NM-TL transition since $\mu=\gamma / v z$ [48]. The obtained value, $z=2.06$, is closer to the estimated for the paramagnetic-KT transition in the XY ( $z=1.84(17)$ [43], $z=1.96(3)$ [49]) and the six-state clock model $(z=1.87(18)[43])$.

Figure 2 presents the $O_{h v}^{2}$ exponent, obtained by fitting the data with Eq. (6), as a function of the temperature. At lower temperatures an increase is observed, with a change in the trend around $T=0.65$, up to a maximum at $T=0.779(5)$ that is very close to the reported temperature for the $h_{2}-\mathrm{NM}$ phase transition. This behavior can be understood by means of the interplay between coarsening and nucleation during the quenching from high temperature to $T<T_{1}$ [47]. In fact, two kinds of relaxation processes have been identified, and they are involved in the system evolution with an occurrence probability that depends on the final temperature. The slowest one corresponds to a coarsening of the NM phase domains followed by nucleation of the $h_{2}$ phase over it, and the other, faster than the previous one, is a direct coarsening to the $h_{2}$ ordered phase. For the former, it was shown that the probability increases with the temperature up to $T_{1}$, while it decreases for the direct $h_{2}$ coarsening process. Hence, for $T>0.65$ the

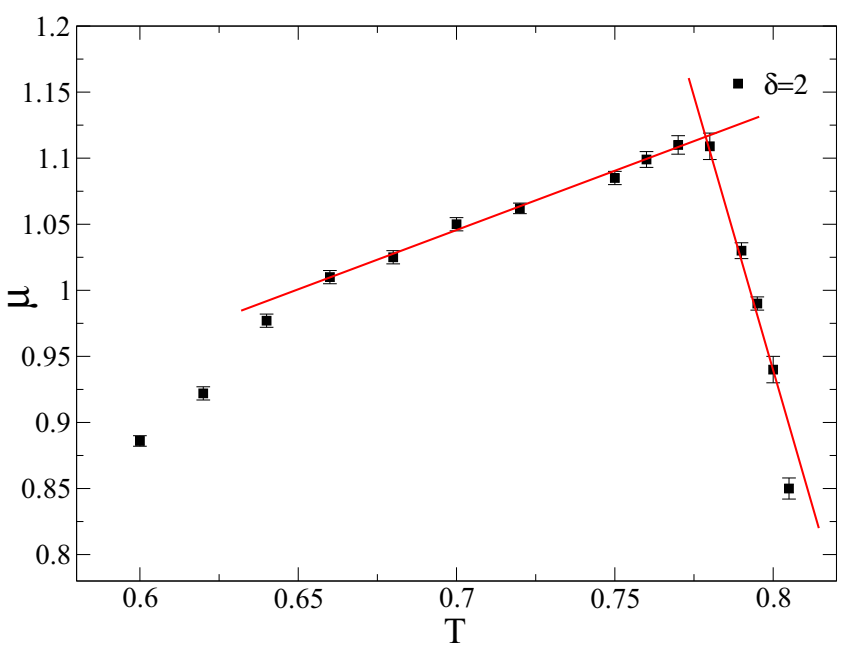

FIG. 2. Dynamic exponent of $O_{h v}^{2}(t)$ as a function of the temperature, corresponding to the paramagnetic initial condition and $\delta=2$. The solid lines are the linear fits used to estimate the maximum value. More details in the text.

coarsening-nucleation process becomes the most probable and its origin may rely on the fact that both phases share the same orientational symmetry [47]. In this way, the acceleration of the ordering dynamics for $T<0.65$ and, consequently, the increase in the $\mu(T)$ exponent, would be related to the fact that the direct coarsening of $h_{2}$ phase is the dominant process and its relaxation time decreases with $T$. In addition, for $T>0.65$ the slow process influences on the dynamics and becomes more relevant with $T$, which would produce a decrease in the slope of $\mu(T)$. Finally, for $T_{1}<T<T_{2}$ the dynamic evolution corresponds to a direct coarsening of the NM phase within the paramagnetic one, as is shown in Figs. 3(a)-3(c). Also, Figs. 3(d)-3(f) shows the expected development of the TL phase at $T=0.83$.

It is worth mentioning that similar results about the temperature dependence of the corresponding order parametermagnetization-exponent, were obtained for the six-state
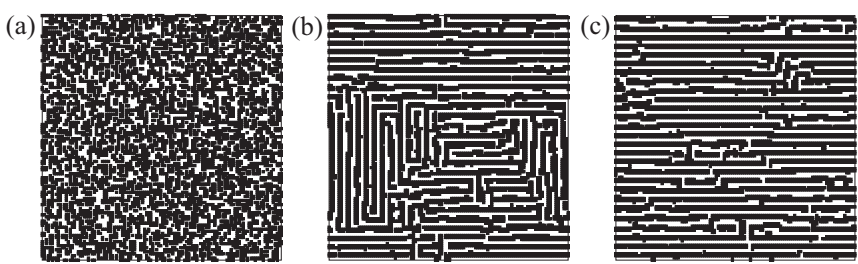

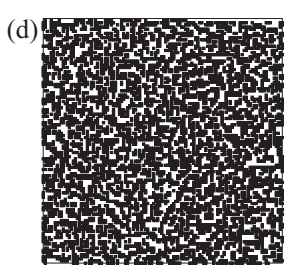

$\mathrm{t}=1 \mathrm{MCS}$

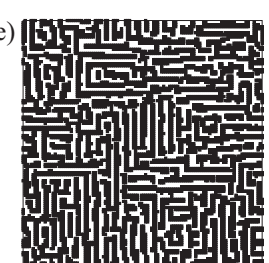

$\mathrm{t}=1 \times 10^{4} \mathrm{MCS}$

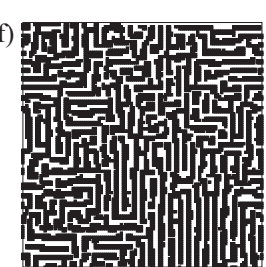

$\mathrm{t}=1 \times 10^{6} \mathrm{MCS}$
FIG. 3. Typical dynamic evolution of the spin configurations during the quenching from high-temperature paramagnetic state to $T=0.79$ [(a)-(c)] and $T=0.83$ [(d)-(f)]. The data correspond to $L=128$ and $\delta=2$. More details in the text. 

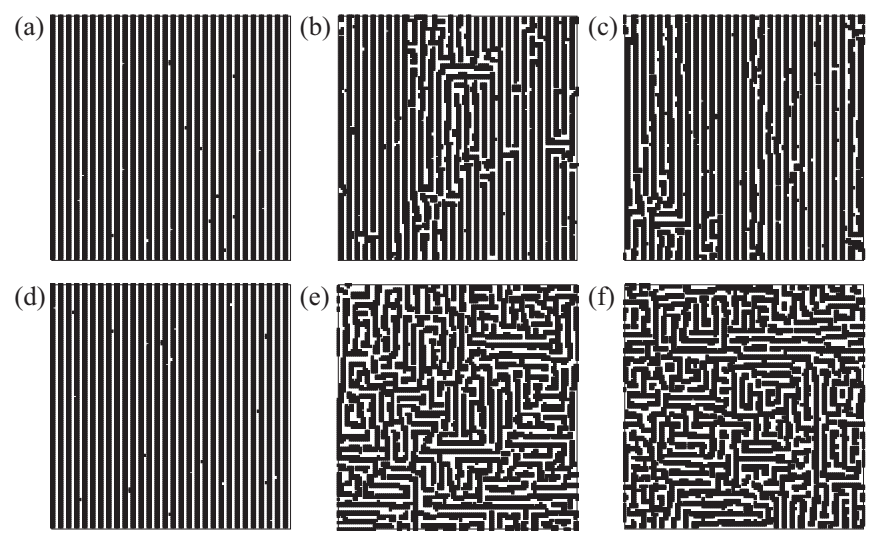

$\mathrm{t}=1 \mathrm{MCS}$

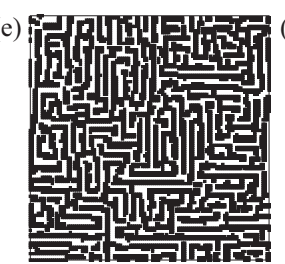

$\mathrm{t}=1 \times 10^{4} \mathrm{MCS}$

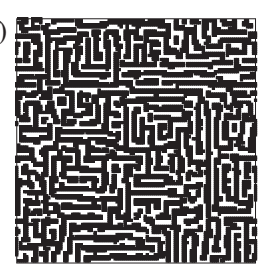

$\mathrm{t}=1 \times 10^{6} \mathrm{MCS}$

FIG. 4. Typical dynamic evolution of the spin configurations during the annealing from ground state to $T=0.79[(\mathrm{a})-(\mathrm{c})]$ and $T=0.83$ [(d)-(f)]. The data correspond to $L=128$ and $\delta=2$. More details in the text.

clock model. This model presents two KT phase transitions, one of them from the high-temperature paramagnetic to a KT phase that exhibits quasi-long-range order and the other one between KT and the ferromagnetic phase [45].

The above results allow us to propose that the STD method is able to detect NM-TL and $h_{2}$-NM phase transitions in the model and to associate the temperature corresponding to the maximum of $\mu(T)$ with $T_{1}$. Moreover, the observation of power laws below $T_{2}=0.805$-also for $T<0.779-$ suggests that both transitions are of the KT type.

In order to confirm these conjectures, the nonequilibrium relaxation from the ground-state initial configuration was simulated at different annealing temperatures. Figure 4 shows a typical dynamic evolution of the spin configurations during the annealing at temperatures $T=0.79$ and $T=0.83$, where the expected NM and TL phases are identified, respectively. Figure 5 shows the time evolution of the $O_{h v}$ at
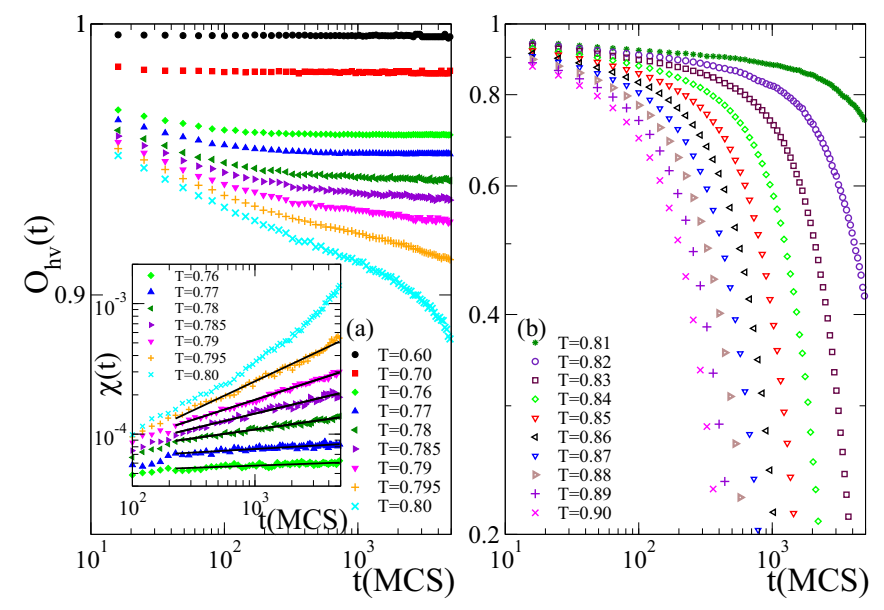

FIG. 5. Nonequilibrium relaxation of $O_{h v}$, corresponding to $\delta=$ 2 , from the ground-state initial configuration at temperatures within the interval (a) $0.60 \leqslant T \leqslant 0.80$ and (b) $0.81 \leqslant T \leqslant 0.90$ from top to bottom in both cases. The inset of figure (a) shows the susceptibility $\chi(t)$ for $0.76 \leqslant T \leqslant 0.80$ (from bottom to top). The continuous lines correspond to power-law fits.

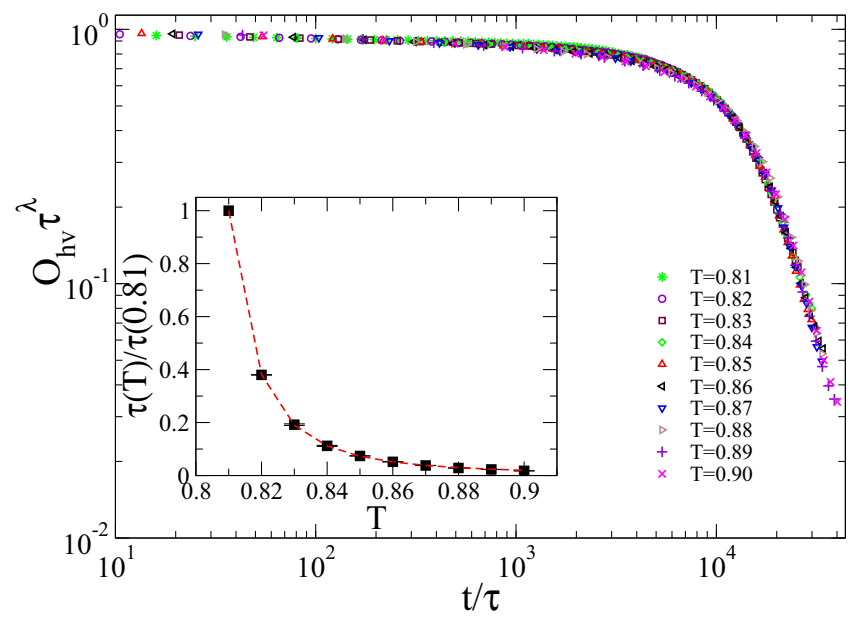

FIG. 6. Scaling of the nonequilibrium relaxation of $O_{h v}$ from the initial ground state, at the temperatures showed in Fig. 5(b), by using Eq. (7) with $\lambda=0.0065$. The inset shows the obtained relaxation time as a function of the temperature. The fit with Eq. (8) is also shown with a dashed line.

several temperatures within the interval $0.60 \leqslant T \leqslant 0.90$. For low temperatures, in the long-time regime, a nonzero value of $O_{h v}$ is observed due to remaining orientational ordering characteristic of the NM phase. This value affects the shorttime evolution of the $O_{h v}$ by introducing departures from the power-law behavior that hinders to distinguish the KT regime, see Fig. 5(a). However, the susceptibility is free of this effect as can be observed in the inset of Fig. 5(a). In fact, this observable diverges as power law at temperatures less than $T=0.80$. This value is very close to that determined for $T_{2}$ from the paramagnetic initial condition, and the behavior at low temperatures also supports the KT-transition conjecture. For $T \geqslant 0.80$ the dynamics of $O_{h v}$ shows the asymptotic exponential decay expected for $T>T_{\mathrm{KT}}$ [39]. This dynamic behavior is not affected by the nonzero value of $O_{h v}$, as consequence the scaling proposed for the KT phase transition by Ozeki et al. [39] can be applied.

Figure 6 displays the scaling of the high-temperature data showed in Fig. 5(b), corresponding to $0.81 \leqslant T \leqslant 0.90$, obtained by means of Eq. (7) with $\lambda=0.0065$ and $v=0.5$. From this scaling, the relaxation time $\tau$ was determined as a function of the temperature (see the inset). The fit of the $\tau(T)$ curve by using Eq. (8) allows us to estimate a KT transition temperature in $T_{\mathrm{KT}}=0.774(2)$. This value is in excellent agreement with that estimated for $T_{1}$ from the $O_{h v}^{2}$ behavior during quenching from the paramagnetic state. This reinforces the idea that the $h_{2}$-NM transition should be of the KT type. Nevertheless, the scaling of the relaxation dynamics does not present signs of the TL-NM transition at $T_{2}=0.805$. This fact may be related to previous results that indicate that free energy barriers were found to be apparently divergent in the thermodynamic limit for $h_{2}$-NM transition but rather small for the NM-TL one $[31,35,47]$. This fact, together with the the small difference between the temperatures corresponding to both transitions, does not allow us to distinguish the dynamic relaxation effects of the NM-TL transition from those of the $h_{2}$-NM one. It is important to note that even if free 


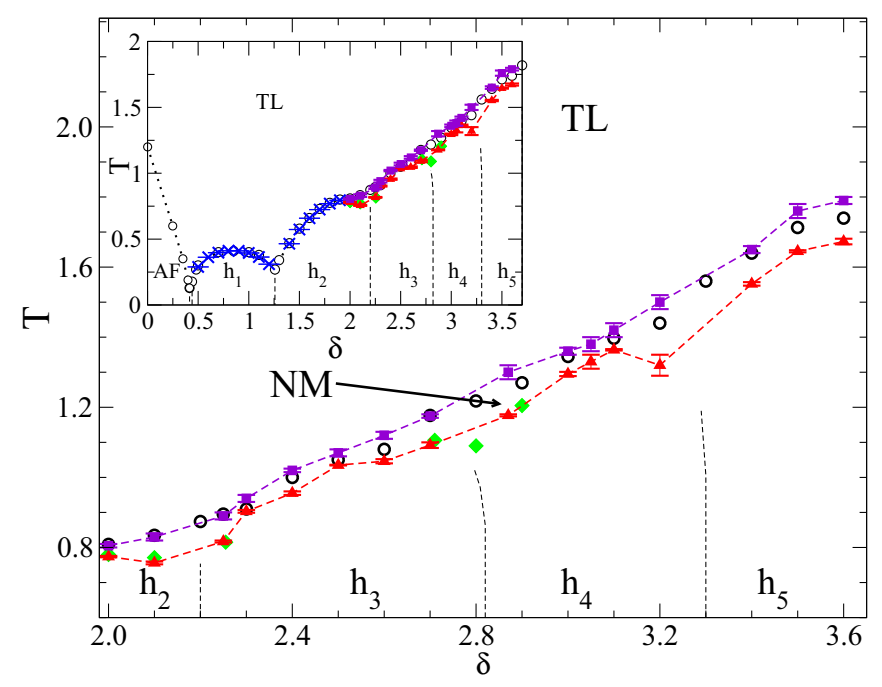

FIG. 7. Phase diagram in the plane $T-\delta$ for $2 \leqslant \delta \leqslant 3.6$. The temperatures $T_{1}$ (triangles) and $T_{2}$ (squares) were obtained by means of the relaxation and STD analysis from the initial $h_{n}$ and paramagnetic states, respectively. Dotted lines are for eye guidance and dashed lines represent the phase boundaries between phases $h_{n}$ and $h_{n+1}$. For the sake of comparison, the data taken from reference [29] are also included: open circles and diamonds refer to the transitions from the high temperature TL to $h_{n}$ (or NM) and NM to $h_{n}$, respectively. The inset shows the whole phase diagram, starting from $\delta=0$, where the crosses correspond to the transition $h_{n}$-TL taken from references [33,34].

energy barriers resemble a first-order phase transition, the specific heat saturation and continuity of internal energy at the thermodynamic limit have been interpreted as evidences of the KT transition phenomenology $[35,47]$. On the other hand, even if $\tau$ can be relatively well fitted by using $\tau=a(\epsilon)^{-b}$-an expression that corresponds to continuous phase transitions

TABLE I. Transition temperatures $T_{1}$ and $T_{2}$ obtained from the STD analysis. The data correspond to the annealing and quenching from two initial conditions, stripes $\left(h_{n}\right)$, and paramagnetic states (DC). The last column presents the estimation of dynamic exponent $z$ corresponding to the NM-TL transition. More details in the text.

\begin{tabular}{llllll}
\hline \hline$\delta$ & $h_{n}$ & $T_{1}\left(h_{n}\right)$ & $T_{1}(\mathrm{DC})$ & $T_{2}(\mathrm{DC})$ & \multicolumn{1}{c}{$z$} \\
\hline 2 & $h_{2}$ & $0.774(2)$ & $0.779(5)$ & $0.805(5)$ & $2.06(2)$ \\
2.1 & $h_{2}$ & $0.756(4)$ & $0.77(1)$ & $0.830(1)$ & $2.19(13)$ \\
2.25 & $h_{3}$ & $0.817(3)$ & $0.83(1)$ & $0.89(1)$ & $2.08(10)$ \\
2.3 & $h_{3}$ & $0.903(4)$ & $0.895(5)$ & $0.93(1)$ & $2.24(11)$ \\
2.4 & $h_{3}$ & $0.955(5)$ & $0.95(1)$ & $1.010(5)$ & $2.5(2)$ \\
2.5 & $h_{3}$ & $1.035(2)$ & $1.032(6)$ & $1.06(1)$ & $2.24(10)$ \\
2.6 & $h_{3}$ & $1.046(6)$ & $1.06(1)$ & $1.12(1)$ & $2.40(16)$ \\
2.7 & $h_{3}$ & $1.092(8)$ & $1.10(1)$ & $1.175(5)$ & $2.13(10)$ \\
2.87 & $h_{4}$ & $1.177(3)$ & $1.18(2)$ & $1.30(2)$ & $2.73(8)$ \\
3 & $h_{4}$ & $1.295(6)$ & $1.29(1)$ & $1.36(1)$ & $2.46(3)$ \\
3.05 & $h_{4}$ & $1.33(2)$ & $1.30(1)$ & $1.38(2)$ & $2.24(3)$ \\
3.1 & $h_{4}$ & $1.364(2)$ & $1.36(1)$ & $1.42(1)$ & $2.40(7)$ \\
3.2 & $h_{4}$ & $1.31(3)$ & $1.38(4)$ & $1.50(2)$ & $3.18(6)$ \\
3.4 & $h_{5}$ & $1.552(9)$ & $1.57(1)$ & $1.65(1)$ & $2.73(17)$ \\
3.5 & $h_{5}$ & $1.645(3)$ & $1.65(1)$ & $1.76(2)$ & $2.11(5)$ \\
3.6 & $h_{5}$ & $1.673(8)$ & $1.69(1)$ & $1.79(1)$ & $2.92(20)$ \\
\hline \hline
\end{tabular}

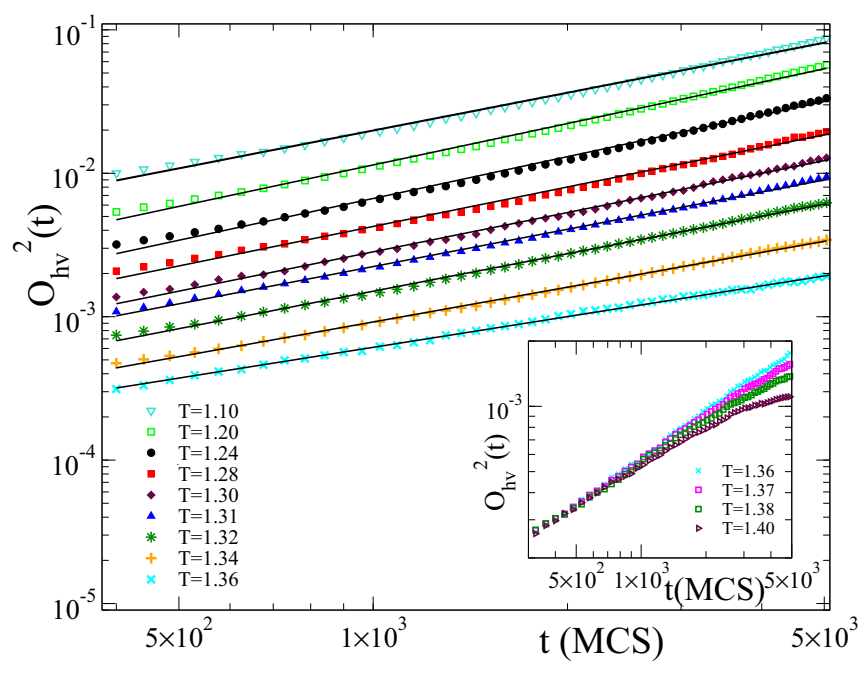

FIG. 8. Log-log plot of the $O_{h v}^{2}$ dynamic evolution from the initial paramagnetic condition at the indicated temperatures for $L=128$ and $\delta=3$. As in the case of $\delta=2$ (see Fig. 1), the data corresponding to different temperatures were shifted by a constant multiplicative factor with the aim of clearly showing their power-law behavior. The inset exhibits the data corresponding to the temperatures at which departures from the power law are observed; for the shake of comparison, $T=1.36$ is also included.

relaxation [42] - it was dismissed due to the power-law divergence of the susceptibility for $T<0.80$ from both initial conditions.

A similar STD behavior was obtained for $\delta=2.1$ case. Indeed, the determined transition temperatures for initial paramagnetic configurations are $T_{1}=0.77(1)$ and $T_{2}=0.83(1)$, which are in good agreement, within error bars, with both $T_{1}=0.756(4)$ obtained from ground-state initial condition and that reported by Pighín and Cannas (see Fig. 7) [29].

Summarizing, even if the orientational order parameter does not seem to be appropriate to characterize the $h_{2}-\mathrm{NM}$ phase transition because both phases present orientational order, the present results for annealing and quenching from ground and paramagnetic states confirm our conjectures about the usefulness of the STD method by employing this observable and its moments in order to determine the $h_{2}$-NM and NM-TL transition points. Moreover, they provide evidence that enables us to conclude that both phase transitions would be of the KT type.

\section{B. Phase transitions for $\delta>2.1$}

It well known that in order to observe the NM phase, the required system sizes must allow the appearance of a large density of defects, such as dislocations and disclinations of stripes [31,35]. In this way, although the presence of NM phase close to the transition lines between $h_{2}-h_{3}$ and $h_{3}-h_{4}$ has already been reported $[29,30]$, it cannot be discarded that this phase may be present in other regions within narrow temperature ranges. Moreover, in these works the finite-size effects due to the small system sizes used- $L \sim 50$ - prevented a clear identification of those regions for $\delta>3$. In this section the STD studies are extended up to $\delta=3.6$, i.e., up to values 


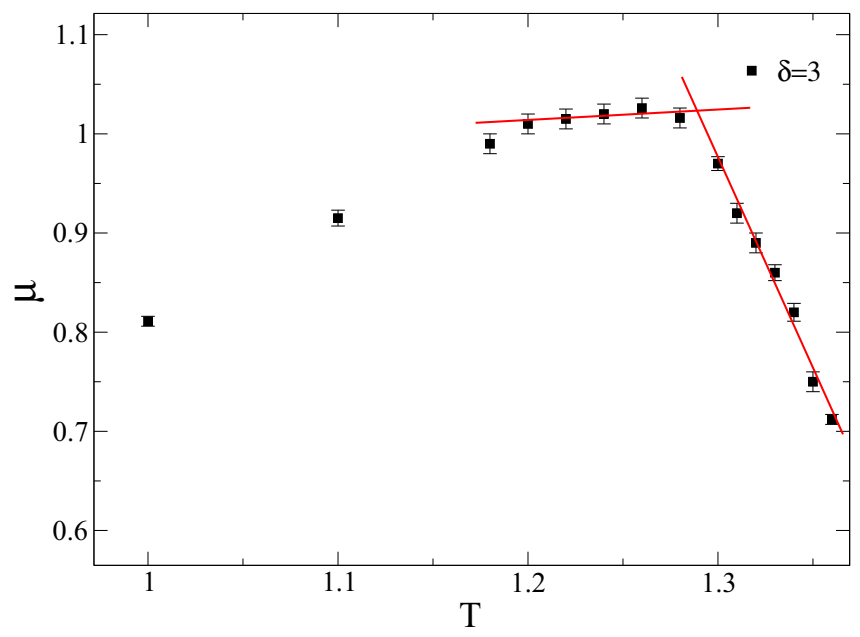

FIG. 9. Dynamic exponent of $O_{h v}^{2}$ as a function of the temperature for the initial paramagnetic condition and $\delta=3$. The solid line corresponds to the linear fits used to determine the maximum value. More details in the text.

where the ground state corresponds to $h_{5}$. For these values of $\delta$, the STD observables present a similar behavior to those described for the $\delta=2$ case. By following the same procedure, the $h_{n}$-NM and NM-TL transition temperatures were estimated and are reported in Table I and Fig. 7. In particular, the cases $\delta=2.25$ and $\delta=2.7$ lead to $T_{1}$ and $T_{2}$ in excellent agreement-within the error bars-with those reported by Pighín and Cannas [29], as shown in Fig. 7. Table I also includes the estimation of the dynamic exponent $z$ corresponding to the NM-TL transition. As can be observed, the obtained values remain closer to those reported for the paramagnetic-KT transition of the $\mathrm{XY}$ and six-state clock models, except at the values of $\delta$ that are near to the $h_{3}-h_{4}$, $h_{4}-h_{5}$ and $h_{5}-h_{6}$ transitions.

Concerning the transitions corresponding to $\delta>2.9$, the case $\delta=3$ was selected to be outlined exhaustively due to the fact that in previous studies no evidence of the NM phase
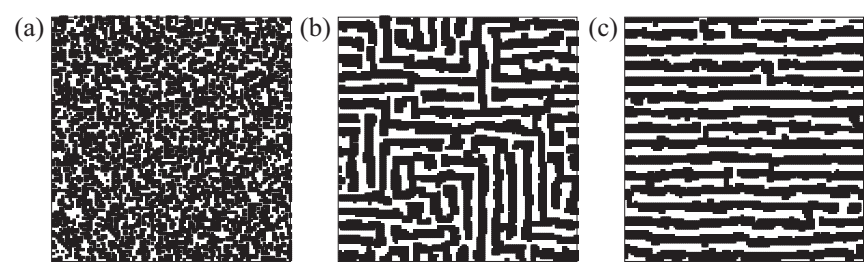

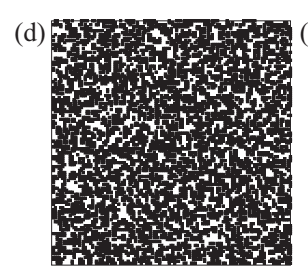

$\mathrm{t}=1 \mathrm{MCS}$

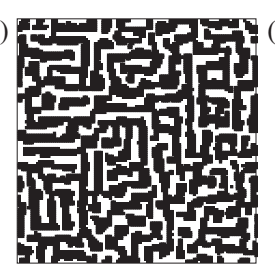

$\mathrm{t}=1 \times 10^{4} \mathrm{MCS}$

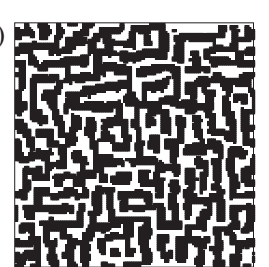

$\mathrm{t}=1 \times 10^{6} \mathrm{MCS}$
FIG. 10. Typical dynamic evolution of the spin configurations during quenching from the high-temperature paramagnetic state to $T=1.315[(\mathrm{a})-(\mathrm{c})]$ and $T=1.50[(\mathrm{~d})-(\mathrm{f})]$. The data correspond to $L=128$ and $\delta=3$. (a)
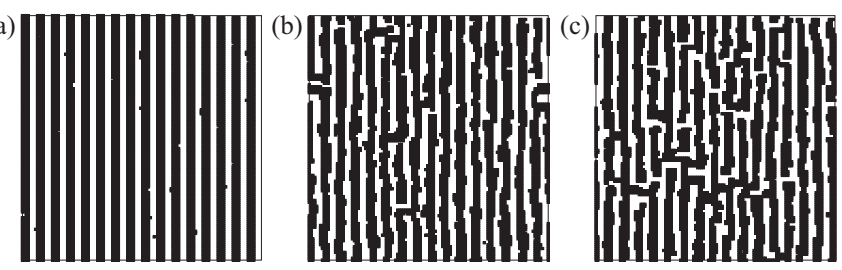

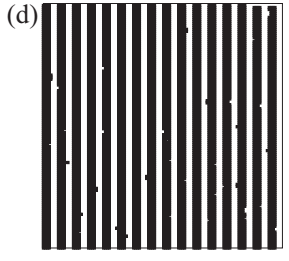

$\mathrm{t}=1 \mathrm{MCS}$

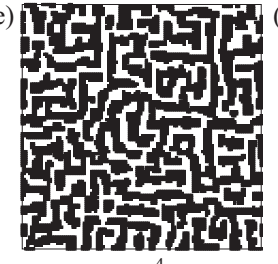

$\mathrm{t}=1 \times 10^{4} \mathrm{MCS}$

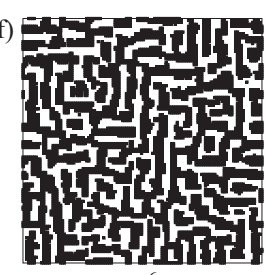

$\mathrm{t}=1 \times 10^{6} \mathrm{MCS}$
FIG. 11. Typical dynamic evolution of the spin configurations during annealing from the ground state to $T=1.315[(\mathrm{a})-(\mathrm{c})]$ and $T=1.50[(\mathrm{~d})-(\mathrm{f})]$. The data correspond to $L=128$ and $\delta=3$.

was found [28,29]. Figure 8 shows that the $O_{h v}^{2}$ dynamic evolution from the initial paramagnetic state at temperatures in the interval $1.10 \leqslant T \leqslant 1.36$ presents a power-law behavior. Meanwhile, the inset exhibits departures from it for $T>1.36$. On the other hand, Fig. 9 displays the $O_{h v}^{2}$ exponent obtained by fitting the data using Eq. (6), which presents a maximum value for $T=1.29(1)$. These results are indicative of the existence of the intermediate NM phase within $1.29<T<1.36$. In order to clear up this point, Fig. 10 follows the dynamic evolution from the paramagnetic state through two series of the snapshots corresponding to $T=1.315$ and $T=1.50$, respectively. As can be observed, for $T=1.315$ a high density of disclinations on the underlying striped structure develops, in concordance with the NM phase, while for $T=1.50$ the TL phase with mutually perpendicular labyrinthine patterns are observed. Moreover, the same configurations are reached when the initial configuration corresponds to the ground state
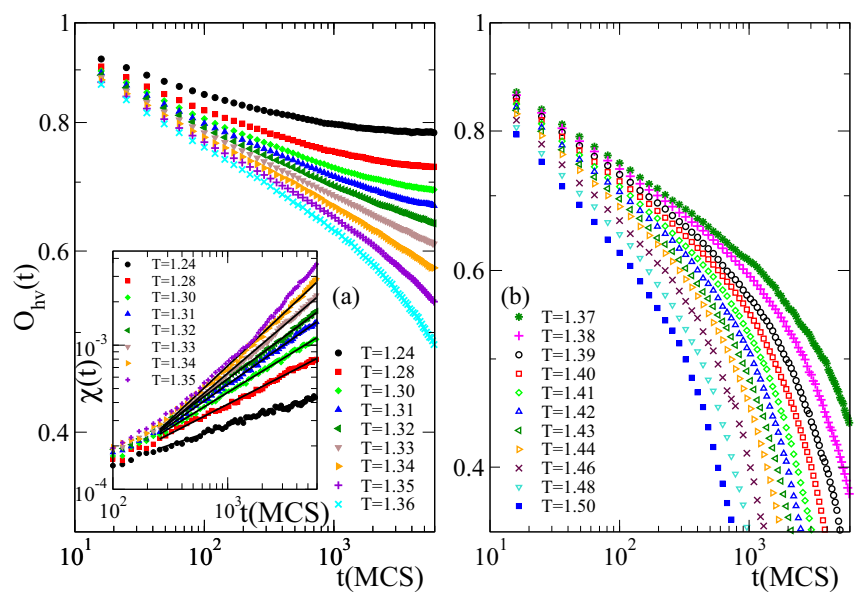

FIG. 12. Nonequilibrium relaxation of $O_{h v}$, corresponding to $\delta=3$,from initial ground state at temperatures within the interval (a) $1.24 \leqslant T \leqslant 1.36$ and (b) $1.37 \leqslant T \leqslant 1.50$ from top to bottom, in both cases. The inset of figure (a) shows the susceptibility $\chi(t)$ for $1.24 \leqslant T \leqslant 1.35$ (from bottom to top). The continuous lines correspond to power-law fits. 


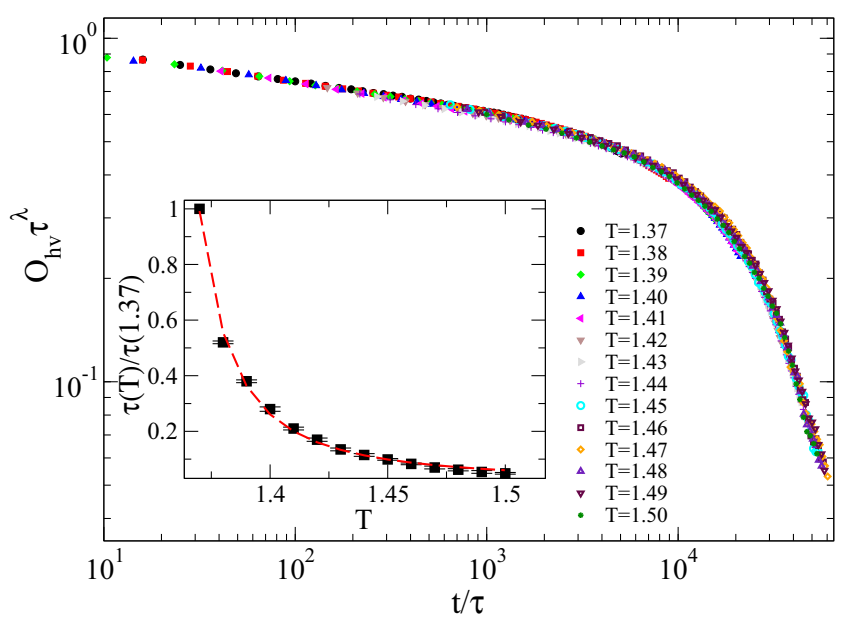

FIG. 13. Scaling of the $O_{h v}$ relaxation from the initial ground state at temperatures within the interval $1.37 \leqslant T \leqslant 1.50$ by using Eq. (7) with $\lambda=0.045$. The inset shows the relaxation time $\tau(T)$ together with the fit with Eq. (8) indicated with a dashed line.

$h_{4}$ (see Fig. 11). In this way, as in the previously studied cases, the STD behavior strongly suggests that both transitions are of the KT type.

Furthermore, the results obtained for the relaxation dynamics from the ground state also support this conjecture. In fact, Fig. 12 presents the $O_{h v}$ time evolution at temperatures in the range $1.24 \leqslant T \leqslant 1.50$, and the inset of Fig. 12(a) shows the power-law divergence of the susceptibility for $T<1.35$, which is close to $T_{2}=1.36(1)$, determined from the paramagnetic initial condition. Again, the $O_{h v}$ power-law departures can be related with a nonzero value of the NM phase in the long-time regime. Figure 13 shows the scaling of the high-temperature data displayed in Fig. 12(b) by using Eq. (7) with $\lambda=0.045$ and $v=0.5$. The inset presents the fit of $\tau(T)$ by means of Eq. (8) that leads us to estimate $T_{\mathrm{KT}}=1.295(6)$ in excellent agreement with $T_{1}$ determined from the annealing.

In this way, the NM phase is expected to be observed for larger values of $\delta$, where the width of the stripes produces more stable configurations with respect to the appearance of topological defects. Our findings also give evidence that the destruction of the long-range positional order is by means of a KT transition, in agreement with the predictions of Abanov et al. [25].

\section{CONCLUSIONS}

Although the ferromagnetic Ising model with dipolar interactions has been the object of intensive research, several points about the phase diagram at zero field remain open to date. In particular, the identification of NM phase has been hampered by strong finite-size effects that are a consequence of the long-range nature of the dipolar interactions. Moreover, the discrepancies reported in the results of the finite-size scaling applied to equilibrium Monte Carlo simulations have prevented the determination of the character of the phase transition from the low-temperature stripe phase $\left(h_{n}\right)$ to NM or TL ones.

In the present work, the study of the short-time dynamic evolution of the orientational order parameter $\left(O_{h v}\right)$ and its moments allowed us to overcome the above-mentioned difficulties. In fact, the power-law behavior of $O_{h v}^{2}$ when the system is quenched from the paramagnetic phase, together with the susceptibility $\chi$ and the scaling of the $O_{h v}$ relaxation during the annealing from $h_{n}$ configuration, indicate the existence of the NM phase along the interval $2 \leqslant \delta \leqslant 3.6$. Moreover, the results suggest that both phase transitions, i.e., $h_{n}$-NM and NM-TL, are of the Kosterlitz-Thouless type. It is also interesting to mention that the transition temperatures estimated by using STD are in excellent agreement with those of the $h_{n}$-NM and NM-TL transitions previously reported for the intervals $2 \leqslant \delta \leqslant 2.25$ and $2.7 \leqslant \delta \leqslant 2.9$ [28,29,31,35]. In conclusion, the findings allow us to establish the existence of the NM phase for $\delta \geqslant 2$ in a narrow temperature range, as was predicted by Abanov et al. [25].

It is important to remark that in this work the short-time dynamics method was successfully applied to study KosterlitzThouless transitions in a model with long-range interactions. This result is important since it can be extended to other models with long-range interactions.

\section{ACKNOWLEDGMENTS}

This work was supported by projects PIP0524-CONICET and X11/784-UNLP, Argentina. We also thank UnCaFiQTINIFTA (SNCAD) for computational resources.
[1] E. Y. Tsymball and D. G. Pettifor, Perspectives of Giant Magnetoresistance in Solid State Physics, Vol. 56, edited by H. Ehrenreich, H. and F. Spaepen (Academic Press, New York, 2001).

[2] E. Y. Tsymball, O. N. Maryasov, and P. R. LeClair, Spindependent tunneling in magnetic tunnel junctions, J. Phys.: Condens. Matter 15, R109 (2003).

[3] B. Hebler, P. Reinhardt, G. L. Katona, O. Hellwig, and M. Albrecht, Double exchange bias in ferrimagnetic heterostructures, Phys. Rev. B 95, 104410 (2017).

[4] U. Edels, L. D. Buda, K. Oumadjela, and P. Wigen, Spin Dynamic in Confined Magnetic Structures I (Springer, Berlin, 2002).
[5] K. Utsumiya, T. Seki, and K. Takanashi, Magnetic properties of 110-FePt/permalloy exchange-spring films, J. Appl. Phys. 110, 103911 (2011).

[6] J. Wei, Z. Zhu, H. Feng, J. Du, Q. Liu, and J. Wang, Top-down control of dynamic anisotropy in permalloy thin films with stripe domains, J. Phys. D: Appl. Phys. 48, 465001 (2015).

[7] T. Dastagir, W. Xu, S. Sinha, H. Wu, Y. Cao, and H. Yu, Tuning the permeability of permalloy films for on-chip inductor applications, Appl. Phys. Lett. 97, 162506 (2010).

[8] R. Lavrijsen, J. H. Lee, A. Fernández-Pacheco, D. C. Petit, R. Mansell, and R. P. Cowburn, Magnetic ratchet for threedimensional spintronic memory and logic, Nature 493, 647 (2013). 
[9] O. Hellwig, A. Berger, J. B. Kortright, and E. E. Fullerton, Domain structure and magnetization reversal of antiferromagnetically coupled perpendicular anisotropy film, J. Magn. Magn. Mater. 319, 13 (2007).

[10] O. Hellwig, A. Berger, and E. E. Fullerton, Magnetic reversal and domain structure in perpendicular AF-coupled films, J. Magn. Magn. Mater. 290-291, 1 (2005).

[11] Y. Shiroishi, K. Fukuda, I. Tagawa, S. Takenoiri, H. Tanaka, H. Mutoh, and N. Yoshicawa, Future options for HDD storage, IEEE Trans. Magn. 45, 3816 (2009).

[12] E. Sallica Leva, R. C. Valente, F. Martínez Tabares, M. Vásquez Mansilla, S. Roshdestwensky, and A. Butera, Magnetic domain crossover in FePt thin films, Phys. Rev. B 82, 144410 (2010).

[13] N. R. Alvarez, J. E. Gómez, M. Vásquez Mansilla, B. Pianciola, D. G. Actis, G. J. Gilardi, L. Leiva, J. Milano, and A. Butera, Magnetic coupling of stripe domains in $\mathrm{FePt} / \mathrm{Ni}_{80} \mathrm{Fe}_{20}$ bilayers, J. Phys. D: Appl Phys. 50, 115001 (2017).

[14] A. Hierro-Rodriguez, R. Cid, M. Vélez, G. RodriguezRodriguez, J. I. Martín, L. M. Álvarez-Prado, and J. M. Alameda, Topological Defects and Misfit Strain in Magnetic Stripe Domains of Lateral Multilayers with Perpendicular Magnetic Anisotropy, Phys. Rev. Lett. 109, 117202 (2012).

[15] K. De'Bell, A. B. MacIsaac, and J. P. Whitehead, Dipolar effects in magnetic thin films and quasi-two-dimensional systems, Rev. Mod. Phys. 72, 225 (2000).

[16] D. P. Pappas, K.-P. Kämper, and H. Hopster, Reversible Transition Between Perpendicular and In-Plane Magnetization in Ultrathin Films, Phys. Rev. Lett. 64, 3179 (1990).

[17] R. Allenspach, M. Stampanoni, and A. Bischof, Magnetic Domains in Thin Epitaxial Co/Au(111) Films, Phys. Rev. Lett. 65, 3344 (1990).

[18] P. Politi, Domain structures in ultrathin magnetic films, Comments Cond. Matter Phys. 18, 191 (1998).

[19] M. Hehn, S. Padovani, K. Ounadjela, and J. P. Bucher, Nanoscale magnetic domain structures in epitaxial cobalt films, Phys. Rev. B 54, 3428 (1996).

[20] A. Vaterlaus, C. Stamm, U. Maier, M. G. Pini, P. Politi, and D. Pescia, Two-Step Disordering of Perpendicularly Magnetized Ultrathin Films, Phys. Rev. Lett. 84, 2247 (2000).

[21] O. Portmann, A. Vaterlaus, and D. Pescia, An inverse transition of magnetic domain patterns in ultrathin films, Nature (London) 422, 701 (2003).

[22] O. Portmann, A. Vaterlaus, and D. Pescia, Observation of Stripe Mobility in a Dipolar Frustrated Ferromagnet, Phys. Rev. Lett. 96, 047212 (2006).

[23] C. A. Ramos, E. Vassallo Brigneti, J. Gómez, and A. Butera, Stripe domains in permalloy films as observed by ferromagnetic resonance and magnetic force microscopy, Physica B (Amsterdam) 404, 2784 (2009).

[24] N. Bergeard, S. Schaffert, V. López-Flores, N. Jaouen, J. Geilhufe, C. M. Günther, M. Schneider, C. Graves, T. Wang, B. Wu, A. Scherz, C. Baumier, R. Delaunay, F. Fortuna, M. Tortarolo, B. Tudu, O. Krupin, M. P. Minitti, J. Robinson, W. F. Schlotter, J. J. Turner, J. Lüning, S. Eisebitt, and C. Boeglin, Irreversible transformation of ferromagnetic ordered stripe domains in single-shot infrared-pump/resonant-x-ray-scatteringprobe experiments, Phys. Rev. B 91, 054416 (2015).
[25] Ar. Abanov, V. Kalatsky, V. L. Pokrovsky, and W. M. Saslow, Phase diagram of ultrathin ferromagnetic films with perpendicular anisotropy, Phys. Rev. B 51, 1023 (1995).

[26] A. B. MacIsaac, J. P. Whitehead, M. C. Robinson, and K. De'Bell, Striped phases in two-dimensional dipolar ferromagnets, Phys. Rev. B 51, 16033 (1995).

[27] A. B. MacIsaac, J. P. Whitehead, K. De'Bell, and K. Sowmya Narayanan, Monte Carlo study of two-dimensional Ising dipolar antiferromagnets as a model for rare-earth ordering in the $\mathrm{R}-\mathrm{Ba}-\mathrm{Cu}-\mathrm{O}$ compounds ( $R=$ rare earth), Phys. Rev. B 46, 6387 (1992).

[28] E. Rastelli, S. Regina, and A. Tassi, Phase transitions in a square Ising model with exchange and dipole interactions, Phys. Rev. B 73, 144418 (2006).

[29] S. A. Pighín and S. A. Cannas, Phase diagram of an Ising model for ultrathin magnetic films: Comparing mean-field and Monte Carlo predictions, Phys. Rev. B 75, 224433 (2007).

[30] E. Rastelli, S. Regina, and A. Tassi, Phase diagram of a square Ising model with exchange and dipole interactions: Monte Carlo simulations, Phys. Rev. B 76, 054438 (2007).

[31] L. G. Rizzi and N. A. Alves, Phase transitions and autocorrelation times in two-dimensional Ising model with dipole interactions, Physica B (Amsterdam) 405, 1571 (2010).

[32] J. S. M. Fonseca, L. G. Rizzi, and N. A. Alves, Stripe-tetragonal phase transition in the two-dimensional Ising model with dipole interactions: Partition function zeros approach, Phys. Rev. E 86, 011103 (2012).

[33] C. M. Horowitz, M. A. Bab, M. Mazzini, M. L. Rubio Puzzo, and G. P. Saracco, Phase transitions and critical phenomena in the two-dimensional Ising model with dipole interactions: A short-time dynamics study, Phys. Rev. E 92, 042127 (2015).

[34] M. A. Bab, C. M. Horowitz, M. L. Rubio Puzzo, and G. P. Saracco, Phase transitions and multicritical behavior in the Ising model with dipolar interactions, Phys. Rev. E 94, 042104 (2016)

[35] S. A. Cannas, M. F. Michelon, D. A. Stariolo, and F. A. Tamarit, Ising nematic phase in ultrathin magnetic films: A Monte Carlo study, Phys. Rev. B 73, 184425 (2006).

[36] Sergio A. Cannas, Daniel A. Stariolo, and Francisco A. Tamarit, Stripe-tetragonal first-order phase transition in ultrathin magnetic films, Phys. Rev. B 69, 092409 (2004).

[37] I. Booth, A. B. MacIsaac, J. P. Whitehead, and K. De'Bell, Domain Structures in Ultrathin Magnetic Films, Phys. Rev. Lett. 75, 950 (1995).

[38] E. V. Albano, M. A. Bab, G. Baglietto, R. A. Borzi, T. S. Grigera, E. S. Loscar, D. E. Rodríguez, M. L. Rubio Puzzo, and G. P. Saracco, Study of phase transitions from short-time non-equilibrium behavior, Rep. Prog. Phys. 74, 026501 (2011).

[39] Y. Ozeki and N. Ito, Nonequilibrium relaxation method, J. Phys. A 40, R149 (2007).

[40] E. S. Loscar, E. E. Ferrero, T. S. Grigera, and S. A. Cannas, Nonequilibrium characterization of spinodal points using short time dynamics, J. Chem. Phys. 131, 024120 (2009).

[41] K. Okano, L. Schülked, K. Yamagishi, and B. Zheng, Monte Carlo simulation of the short-time behavior of the dynamic XYmodel, J. Phys. A 30, 4527 (1997).

[42] Y. Echinaka and Y. Ozeki, Improved dynamical scaling analysis using the kernel method for nonequilibrium relaxation, Phys. Rev. E 94, 043312 (2016). 
[43] Y. Ozeki, K. Ogawa, and N. Ito, Nonequilibrium relaxation analysis of Kosterlitz-Thouless phase transition, Phys. Rev. E 67, 026702 (2003)

[44] H. J. Luo, L. Schülke, and B. Zheng, Dynamic Approach to the Fully Frustrated XY Model, Phys. Rev. Lett. 81, 180 (1998).

[45] P. Czerner and U. Ritschel, Universal short-time dynamics in the Kosterlitz-Thouless phase, Phys. Rev. E 53, 3333 (1996).

[46] D. E. Rodriguez, M. A. Bab, and E. V. Albano, Study of the nonequilibrium critical quenching and the annealing dynamics for the long-range Ising model in one dimension, J. Stat. Mech.: Theory and Experiment (2011) P09007.

[47] S. A. Cannas, M. F. Michelon, D. A. Stariolo, and F. A. Tamarit, Interplay between coarsening and nucleation in an Ising model with dipolar interactions, Phys. Rev. E 78, 051602 (2008).

[48] B. Zheng, M. Schulz, and S. Trimper, Dynamic simulations of the Kosterlitz-Thouless phase transition, Phys. Rev. E 59, R1351 (1999).

[49] B. Zheng, Monte Carlo simulations of short-time critical dynamics, Int. J. Mod. Phys. B 12, 1419 (1998). 